This item was submitted to Loughborough's Research Repository by the author.

Items in Figshare are protected by copyright, with all rights reserved, unless otherwise indicated.

\title{
Excimer laser machining of microvias in glass substrates for the manufacture of high density interconnects
}

PLEASE CITE THE PUBLISHED VERSION

http://dx.doi.org/10.1007/s00340-012-5106-6

PUBLISHER

(C) Springer Verlag

VERSION

AM (Accepted Manuscript)

LICENCE

CC BY-NC-ND 4.0

REPOSITORY RECORD

Bhatt, Deepa, David A. Hutt, and Paul P. Conway. 2019. "Excimer Laser Machining of Microvias in Glass Substrates for the Manufacture of High Density Interconnects". figshare. https://hdl.handle.net/2134/13359. 
This item was submitted to Loughborough's Institutional Repository (https://dspace.lboro.ac.uk/) by the author and is made available under the following Creative Commons Licence conditions.

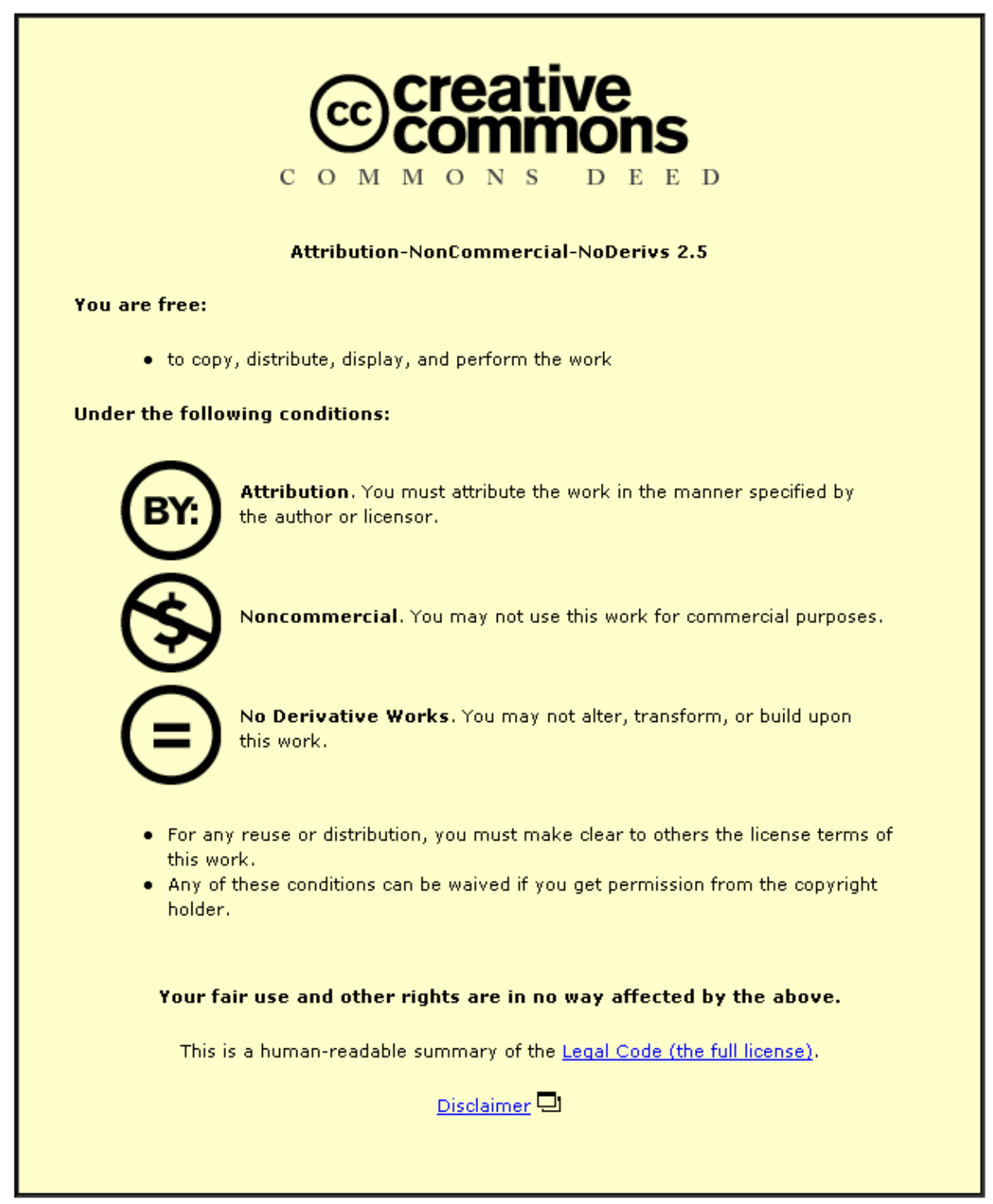

For the full text of this licence, please go to: http://creativecommons.org/licenses/by-nc-nd/2.5/ 


\title{
Excimer laser machining of microvias in glass substrates for the manufacture of high density interconnects
}

\author{
Deepa Bhatt ${ }^{1}$, David A. Hutt ${ }^{2}$, Paul P. Conway ${ }^{2}$ \\ ${ }^{1}$ Wolfson School of Mechanical and Manufacturing Engineering, Loughborough University, \\ Loughborough, LE11 3TU, U.K. \\ Email: d.bhatt74@gmail.com \\ ${ }^{2}$ Wolfson School of Mechanical and Manufacturing Engineering, Loughborough University, \\ Loughborough, LE11 3TU, U.K. \\ Email: d.a.hutt@lboro.ac.uk
}

\begin{abstract}
Machining of microvias in 100-50 $\mu$ m thick CMZ glass using an excimer laser (248 nm) was investigated. The effect of various laser process parameters: pulse energy, repetition rate, irradiation time were studied to optimise the microvia drilling process and a process window was identified. Through-hole drilling of $100 \mu \mathrm{m}$ diameter (entry hole) microvias was achieved at a fluence (energy density) as low as $2.3 \mathrm{~J} / \mathrm{cm}^{2}$ with an irradiation time of 30-40 s at a repetition rate of $20 \mathrm{~Hz}$, giving a taper angle between $22-24^{\circ}$ relative to the vertical. However, by increasing the fluence to 4.5 $\mathrm{J} / \mathrm{cm}^{2}$, this reduced the machining time to 5-10 s and taper angle to $14^{\circ}$, giving an exit hole diameter of around 45-50 $\mu \mathrm{m}$. With $50 \mu \mathrm{m}$ thick glass, it was possible to machine through-hole microvias with smaller entry hole diameters down to $40 \mu \mathrm{m}$. Machined microvias were characterised to investigate debris, recast layer and microcrack formation. Debris and recast layer around the machined features was minimised by using a protective photoresist layer coating on the glass and through appropriate operating parameter selection. Microcracks along the sidewalls of the microvias could not be avoided, but their severity depended on the laser machining parameters used.
\end{abstract}




\section{Introduction}

New technologies and processes are being developed to enable manufacturers to meet the growing demand for miniaturised electronic devices [1]. One way in which this is achieved is through the miniaturisation of the components, e.g. integrated circuits, and the minimisation, or removal, of the packaging that they are housed within. Flip-chip interconnection technology, where the bare semiconductor device is connected directly to the circuit board (substrate) is one approach to reduce the size of products and is being widely used for electronic and opto-electronic devices as it offers one of the smallest component footprints [2,3,4]. However, to match the reductions in component size, similar reductions in the size of features of the substrates to which they are attached is required. High density interconnection enables electronic devices to be assembled in the minimum amount of space by reducing track widths and pitch sizes, and by using microvias (through hole and blind) to link different planes within multilayer circuit boards. However, manufacturing multilayer substrates for flip chip devices from conventional organic materials such as FR-4 (glass fibre reinforced epoxy) suffers from low production efficiency and inservice reliability, due to their poor dimensional stability (unpredictable expansion and contraction during manufacture) and high thermal expansion coefficient (causes thermal mismatch between substrate and semiconducter chip) [5,6]. Research is therefore underway to investigate the use of thin sheets of glass (50-100 $\mu \mathrm{m}$ thick) which has the potential for building multilayer substrates $[7,8]$. Glass offers various advantages such as: good dimensional stability that makes it possible to machine fine features accurately; low thermal expansion coefficient which closely matches with silicon and enables reduced thermo-mechanical stresses in flip-chip solder joints; and 
high optical transparency to assist in the alignment of microvias with buried features in multilayer substrates.

High density interconnections demand manufacturing processes which can machine microvias and tracks with diameters and widths down to $50 \mu \mathrm{m}$ with a high level of accuracy. Currently, three major technologies: photo-imaging, plasma etching and laser drilling are commercially used to create microvias in organic substrate materials [9]. However, laser micromachining is a more appropriate process to produce features in glass [10]. In the electronics industry, for the manufacture of FR-4 substrates, $\mathrm{CO}_{2}$ and Nd:YAG lasers are commonly used for the drilling of microvias as they are able to efficiently remove the polymer and glass fibres. However, for the machining of hard materials like glass and ceramics, excimer lasers are more appropriate since they can operate in the ultraviolet range (193 nm - $353 \mathrm{~nm}$ ) [11]. Also, since the excimer laser uses the mask projection technique, it is possible to machine features with a wide range of shapes down to $10 \mu \mathrm{m}$ in size with a high level of accuracy, which makes it a popular tool in the optoelectronics and microelectronics industries. The short pulse duration, high fluence and high pulse energy allows the radiation to be efficiently absorbed by the surface of most materials [12]. However, while working with brittle materials, such as glass, an important consideration is that there must be no flaws or stress raising factors, such as debris, that can initiate microcracks and further, may affect other manufacturing processes and ultimately limit the reliability of the device. For electrical interconnects, microvias with no debris and walls without undercuts are required $[9,13]$. 
This paper presents the results of an investigation of the micromachining of 50 and $100 \mu \mathrm{m}$ thick CMZ glass sheets with a $\mathrm{KrF}$ excimer laser (248 nm) using a mask projection technique to produce microvias. In this work CMZ glass, which is a borosilicate type supplied by Qioptiq [14], was chosen due to its close CTE match to Si and good availability in thin sheets, making it suitable for the potential application. The KrF excimer laser was found to be suitable for this work as spectrophotometric measurements showed good absorption at this wavelength. The effect of the individual laser parameters on the machining of glass was studied to optimize the process, after which machined features were characterized using SEM and optical microscopes to investigate the formation of debris and recast layers around the microvias and the occurrence of microcracks.

\section{Experimental details}

A KrF Excimer laser (203 Lambda Physik model) operating at $248 \mathrm{~nm}$ was used with a specification of $400 \mathrm{~mJ}$ maximum output pulse energy, $34 \mathrm{~ns}$ pulse length, an average power of $100 \mathrm{Watt}$ and $200 \mathrm{~Hz}$ maximum repetition rate. The beam exiting the laser unit was inhomogeneous and rectangular in shape (22 $\mathrm{mm} \times 6 \mathrm{~mm}$ ). To improve the beam profile it was passed through the optics train consisting of vertical (LV1-LV2) and horizontal (LH1-LH2) cylindrical lenses as shown in figure 1 to fold the beam and make it parallel with a square cross section in the vertical and horizontal directions. A scanning mirror (Mirror 3) could be used to scan the beam across the mask plane to produce a more homogeneous beam intensity distribution at the workpiece. However, for drilling microvias of around $100 \mu \mathrm{m}$ diameter entry hole or less, the use of the scanning mirror did not offer any significant change in microvia machining rate and quality and hence this was not used in most of the experiments 
during microvia machining, however it was used for other larger sized features $(\geq 200$ $\mu \mathrm{m})$ such as grooves and tracks. The beam was passed through the mask plane / aperture with a maximum area of $15 \times 15 \mathrm{~mm}^{2}$ before going through a projection lens that gave 15 times of linear reduction at the workpiece and a maximum spot size of 1 $\mathrm{mm}^{2}$. The mask placed in the beam path was used to tailor the shape and size of the beam spot delivered to the workpiece. For this particular study, different sized circular masks fabricated with two different methods: mechanical drilling and chemical etching, were used to produce microvias in the CMZ glass. The workpiece rested on the XYZ CNC table. The $\mathrm{X}$ and $\mathrm{Y}$ axis tables with a resolution of $0.5 \mu \mathrm{m}$ and $1 \mu \mathrm{m}$ respectively were used for lateral movement of the workpiece during machining, while the $\mathrm{Z}$ axis table with a resolution of $0.01 \mu \mathrm{m}$ was used to position the sample in the vertical direction to achieve fine focus of the laser beam on the workpiece. Focus control was achieved both manually and automatically with the help of a diode laser and photodiode array detector. The diode laser beam was reflected from the workpiece surface (at the working position) to a photodiode array detector which provided positional measurement. For machining trials, the pulse energy at the workpiece was measured using a power meter.

Microvias were machined in CMZ glass sheets primarily $100 \mu \mathrm{m}$ (with a specification of $\pm 15 \mu \mathrm{m}$ ) thick and, in some cases, $50 \mu \mathrm{m}$ thick, supplied by Qioptiq. Through-hole microvias of $100 \mu \mathrm{m}$ diameter entry hole were machined to characterize the effect of variations in fluence $\left(\mathrm{J} / \mathrm{cm}^{2}\right)$, pulse repetition rate $(\mathrm{Hz})$ and irradiation time to identify the process window for machining microvias. Further, to explore the process limits, different diameter microvias were drilled to identify the smallest size capable with the laser set-up. The machined glass samples were cleaned in an 
ultrasonic bath with iso-propanol to remove the loose debris and samples shown here have all undergone this process unless otherwise stated. Machined microvias were characterized using an Olympus optical microscope and SEM (Leo S360). For ablation depth and sidewall surface roughness measurement, a Zygo White light interferometer and Talysurf CLI 2000 non-contact laser gauge were used. The diameter of entry and exit holes were measured from optical microscope images using a calibrated scale. The variation in the diameters of holes presented here should therefore be considered as $\pm 3 \mu \mathrm{m}$.

\section{Results and Discussion}

\subsection{Threshold Fluence}

Since glass is a strongly bonded material, it requires high laser fluence for ablation compared to other polymeric and metallic materials [15]. An ablation threshold of around $1.5 \mathrm{~J} / \mathrm{cm}^{2}$ for the CMZ glass was obtained by increasing the laser beam energy $(\mathrm{mJ})$ per pulse at the workpiece, measured with a power meter, and noting when machining of the sample commenced. The ablation threshold was also obtained from other experimental values, by extrapolating a plot of ablation depth as a function of fluence on a semi-log scale and this was found to be in close agreement with the above measured value.

\subsection{Process Optimisation}

Microvias were machined in $100 \mu \mathrm{m}$ thick CMZ glass using various circular mask apertures fabricated by different techniques. The shape and size of the entry holes for microvias drilled in the CMZ glass were found to be in close agreement with 
those expected from the geometry of the mask. Using mechanical drilling to create the mask in a steel sheet led to holes with an irregular shape that was reproduced in the shape of the microvias that were around $100 \mu \mathrm{m}$ in diameter. Using photolithography and etching to produce a brass mask gave highly circular apertures with correspondingly circular vias of $93 \mu \mathrm{m}$ diameter.

To identify the process window for microvia drilling, the effect of laser operating parameters: laser pulse energy / energy fluence (energy per pulse for unit area of the beam spot size), repetition rate and irradiation time, were investigated. Through hole drilling of $100 \mu \mathrm{m}$ diameter microvias in $100 \mu \mathrm{m}$ thick glass was successfully achieved at energy fluence as low as $\sim 2.3 \mathrm{~J} / \mathrm{cm}^{2}$ at $30 \mathrm{~Hz}$ repetition rate and $40 \mathrm{~s}$ irradiation time. However, a tapered profile was identified in all the microvias irrespective of the size. Figure 2 shows the cross section of a $100 \mu \mathrm{m}$ diameter microvia drilled in $100 \mu \mathrm{m}$ thick glass using $4 \mathrm{~J} / \mathrm{cm}^{2}$ with a tapered profile across the thickness and a taper angle, measured from the vertical, of around $19^{\circ}$.

The taper on the holes limited the exit hole diameter that could be achieved and subsequently would restrict the minimum size of entry hole. This in-turn, would impact on the pitch of the interconnect that could be created in any circuit board. Part of the investigation therefore considered how the taper of the holes could be reduced and to monitor this, the effect of process parameters on the exit hole size was considered. Figure 3 shows a series of graphs relating exit hole diameter to laser machining parameters for $100 \mu \mathrm{m}$ diameter entry hole microvias. It should be noted that in all of these graphs, an exit hole diameter of $0 \mu \mathrm{m}$ corresponds to the situation where no exit hole was observed (no through hole was drilled) and the trend line 
joining these points to the subsequent data should be considered as a guide to the eye only.

Increasing energy fluence had a great impact on the microvia drilling process. Figure 3a shows the plot for exit hole diameter as a function of machining time for different fluence and repetition rate. With increase in energy fluence, the exit hole diameter of the microvia increased. The maximum fluence achieved at the workpiece with the standard beam delivery set-up was $\sim 3 \mathrm{~J} / \mathrm{cm}^{2}$ and this limited the size of the exit hole diameter to around $30 \mu \mathrm{m}$ with a corresponding minimum taper angle of $19^{\circ}$. As can be seen from Figure 3a, increasing the machining time initially led to an increase in the exit hole size, but after a period of time, extended exposure did not lead to any further changes. Increasing the repetition rate for the same fluence did not increase the exit hole significantly.

Since fluence was a major driving force to increase the size of the exit hole of the microvia, further efforts were made to increase the fluence at the workpiece. To achieve this, the attenuator, one of the optical elements in the beam delivery system, was removed, as a result of which, the maximum fluence at the workpiece increased from $3 \mathrm{~J} / \mathrm{cm}^{2}$ to $4.5 \mathrm{~J} / \mathrm{cm}^{2}$. Figure $3 \mathrm{~b}$ shows the effect of this change on the exit hole size as a function of repetition rate for fixed time. At $4.5 \mathrm{~J} / \mathrm{cm}^{2}$ the taper angle reduced to $14^{\circ}$ and the maximum achievable exit hole diameter increased to around $45-50 \mu \mathrm{m}$.

As shown in figures $3 a$ and $b$, increasing the irradiation time or repetition rate initially increased the exit hole size, but this then reached a plateau where the taper angle did not change, although as will be described later the amount of debris and 
recast layer formation did increase. This means that the performance of the microvia drilling process is highly influenced by the number of pulses delivered to the sample (the number of pulses is obtained by multiplying repetition rate and irradiation time). Figure 3c shows plots of the exit hole diameter as a function of the number of pulses delivered for $100 \mu \mathrm{m}$ entry holes for two different fluences of $4.5 \mathrm{~J} / \mathrm{cm}^{2}$ and $3 \mathrm{~J} / \mathrm{cm}^{2}$. These were obtained using different combinations of frequencies and irradiation times. It can be seen from the plot that through-hole drilling commenced after delivering a fixed number of pulses depending on the fluence. A minimum of 150 pulses were required even at the maximum fluence of $4.5 \mathrm{~J} / \mathrm{cm}^{2}$. The exit hole diameter increased with increasing number of pulses up to a limit depending on the fluence, beyond which no significant increase in the size of the exit hole diameter was observed. It is apparent from the data that the exit hole diameter was not influenced by the combination of frequency and machining time used to form the microvias i.e. a lower pulse frequency delivered for a longer time, gave similar results to a higher pulse frequency for less time.

During the microvia drilling process, diffractive effects at the edge of the hole are likely to be produced, which may cause low fluence and low ablation rates near the edges of the hole compared to the centre of the hole. Such effects produce the tapered holes with the progression of drilling through the thickness of the glass as observed here [16]. The edge effect is expected to decrease with increase in fluence and so the taper angle should also decrease. Figure 3d shows the taper angle of the microvias as a function of the fluence and shows a largely linear decrease in the taper angle with increase in fluence, in agreement with the above statement. Other factors such as the numerical aperture of the optics, beam divergence and shadowing effects 
also favour a tapered profile of the holes. This means that along with laser fluence, the optical set-up of the laser system also plays an important role in the final taper profile achieved [17]

Due to the tapered profile, through-hole microvia drilling was restricted to a minimum entry hole size depending on the glass thickness used, below which it was difficult to achieve through-holes. To explore this limit, different entry hole size microvias from $100 \mu \mathrm{m}$ to $25 \mu \mathrm{m}$ in diameter were drilled in $50 \mu \mathrm{m}$ thick CMZ glass. Through-hole drilling was successfully achieved down to $40 \mu \mathrm{m}$ entry hole size, but beyond this, only blind microvias were formed. Based on the above practical limitations, a final aspect ratio of the order of 1:1.25 (entry hole diameter to thickness) was the best that could be achieved in CMZ glass with the excimer laser used here.

\subsection{Microvia Characterisation}

For electrical substrate manufacture, it is necessary to produce clean, defect free microvias with precise feature size and minimal sidewall roughness for reliable interconnection. An irregular sidewall and cross section profile of the microvia can cause void formation during metallisation, subsequently leading to failure of the device. Straight or inclined profiles enable uniform smooth metal coating of microvias [13]. As discussed in the previous section, a wide operating window was identified for microvia drilling in CMZ glass, which produced microvias with a tapered profile and with different size and shape of the exit hole. However debris, recast layer and microcrack formation were the key issues during machining which are discussed in this section. 
While machining microvias in CMZ glass, a loose debris and recast layer were formed around the entry and exit holes. To remove this, glass samples were ultrasonically agitated in an iso-propanol bath. Loose debris around the microvias were easily removed by this method, however it was difficult to completely remove the hard crust of recast layer. Figures $4 a$ and $b$ show SEM images of microvias before and after cleaning in this way.

The quality of the microvias was strongly influenced by process parameters. This is generally expected since the laser - material interaction theory is very complex and it has been described by several authors that excimer laser ablation in glass takes place with both athermal (material removal occurs by direct breaking of bonds) and thermal (material removal occurs by heating, melting and ejection) mechanisms depending on the wavelength and process parameters [17-19]. Since a recast layer around the entry and exit holes was observed here, it can be assumed that at least some of the material was removed by thermal ablation. Figure $5 \mathrm{a}$ and $\mathrm{b}$ compares SEM images of microvias drilled at high and low fluences of $4.2 \mathrm{~J} / \mathrm{cm}^{2}$ and $2.6 \mathrm{~J} / \mathrm{cm}^{2}$. The microvia drilled at $4.2 \mathrm{~J} / \mathrm{cm}^{2}$ gave a bigger exit hole $\sim 40 \mu \mathrm{m}$, the shape of which closely matched with that of the entry hole. In the microvias drilled at $2.6 \mathrm{~J} / \mathrm{cm}^{2}$, the exit holes were very small with irregular geometry and the edge quality of the holes, especially near the entry holes was poor. This is thought to be because, as described by Hornberger et al. [19], at lower fluence, the heating and melting effect dominates, as absorbed energy is not enough to cause breaking of bonds, but capable of promoting lattice vibration, which means that thermal ablation predominates at low fluence. During microvia drilling a dense plasma cloud of an ionised material is likely to form which may absorb part of the beam energy resulting in less energy reaching 
the target and causing thermal damage around the holes $[15,19,20]$. Moreover, as described by Bogaerts et al. [21], a nanosecond pulse duration, such as 34 ns used here, causes some energy loss by thermal dissipation and so less energy is available for the ablation.

In order to reduce the level of debris and recast layer adhering to the surface around the microvias, a protective polymer film (in this case, a dry-film photoresist) was applied on the glass surface before machining so that during laser ablation debris and recast layer would attach to the film which could then be removed leaving a clean surface. This technique is quite similar to that used by Kawamura et al. [22]. This approach minimized the amount of debris and recast layer adhering to the glass, but it was still difficult to prevent it completely. It was observed that, with a high number of pulses, it was still difficult to prevent the deposition of debris around the microvia. Figures $6 a$ and $b$ show optical images of the entry holes of microvias drilled in glass that was first laminated with a photoresist film and then machined using $4.5 \mathrm{~J} / \mathrm{cm}^{2}$ fluence and different numbers of pulses. The microvia drilled with 1600 pulses showed a greater amount of deposited recast layer. As discussed in the previous sections, an increase in the number of pulses, increased the exit hole diameter of microvias up to a particular level depending on the fluence, after which no significant increase was observed. From this it can be predicted that further exposure of the laser beam or increase in number of pulses dissipated the pulse energy around the machined feature causing heating and melting around the entry and exit holes and formed thick deposits of recast layer [23]. Since the ablation threshold of the photoresist material was much lower than that of the CMZ glass, heat dissipated in the surrounding area during machining was enough to vaporise the photoresist film 
and so the debris and recast layer adhered to the glass surface. The use of the photoresist layer was very effective for $100 \mu \mathrm{m}$ diameter microvias drilled in the 50 $\mu \mathrm{m}$ thick glass, which showed clean microvias with almost negligible debris adhered (figure 6c). This method was useful not only to reduce the level of debris, but also for the subsequent metallisation process which enabled selective plating on the laser machined glass [7].

It was observed earlier that the exit hole diameter depended on the total number of pulses delivered, irrespective of the repetition rates and irradiation times used. However, a wide variation in surface morphology and geometry of the exit hole was observed for the same number of pulses delivered with different combinations of repetition rates and irradiation times. Figure 7 shows the optical microscope images of entry and exit holes of $100 \mu \mathrm{m}$ diameter microvias drilled in $100 \mu \mathrm{m}$ thick CMZ glass for three different drilling times: $5 \mathrm{~s}, 10 \mathrm{~s}$ and $20 \mathrm{~s}$, at $4.5 \mathrm{~J} / \mathrm{cm}^{2}$ fluence and 60 $\mathrm{Hz}$ repetition rate. The exit hole size increased a little with drilling time from $5 \mathrm{~s}$ to $10 \mathrm{~s}$ and then remained almost the same around $50 \mu \mathrm{m}$ for $20 \mathrm{~s}$. However, despite having the same size, in the latter case, a greater amount of thermal damage around the hole was found. While examining glass machined with microvias, the presence of a Heat Affected Zone (area around the machined feature in which material properties are altered from the bulk material) was observed around the entry and exit holes, especially for those exposed for long irradiation times. This was identified using an optical microscope as a discoloured region around the entry and exit holes as shown in figure 7f. As discussed above, after a certain point, an increase in the number of pulses caused the pulse energy to dissipate in the surrounding area of the machined feature, which is likely to alter the glass properties and form a heat affected zone 
(HAZ) in that region. The width of the HAZ appeared to depend on the laser machining parameters and laser pulse duration. At low energy fluence of around 2-3 $\mathrm{J} / \mathrm{cm}^{2}$ with the minimum required pulses to drill the maximum achievable exit hole of the microvia, the HAZ was larger in area compared to that observed for a higher energy fluence of $4.5 \mathrm{~J} / \mathrm{cm}^{2}$. Furthermore, increasing the number of pulses or irradiation time beyond that required to achieve the maximum exit hole diameter, also increased the size of the HAZ. The existence of a HAZ around laser machined features in glass has been reported by several authors in the literature $[17,19,24]$. Detailed investigation and characterisation of the HAZ is still required however, to fully understand this behaviour and its effect on the glass.

Although at lower irradiation time the exit and entry holes had less debris and recast layer around them, the sidewalls of the microvia interiors were found to be rough with uneven material removal. Figure 8 shows SEM images of $100 \mu \mathrm{m}$ microvias where uneven removal of material along the sidewalls was identified in the microvia drilled at $5 \mathrm{~s}$, but with increase in irradiation time to $20 \mathrm{~s}$, uniformity along the side wall surface increased.

As mentioned previously, the sidewall roughness of the microvia is an important factor for the subsequent metallisation process and reliable interconnection. Sidewall surface roughness of the laser machined microvias was measured using two techniques: Zygo white light interferometer and Talysurf CLI 2000. CMZ glass samples machined with microvias were carefully scribed using a diamond pen, which allowed the glass to be broken across the microvia exposing the interior surface similar to that shown in figure 2. Roughness values were measured in two microvias 
drilled at $30 \mathrm{~Hz}$ and $60 \mathrm{~Hz}$ with a fixed fluence of $4.5 \mathrm{~J} / \mathrm{cm}^{2}$ for $20 \mathrm{~s}$. Roughness of the sidewalls was measured in three different regions along the thickness of the glass across the microvia cross section: near the entry hole (top), in the central region (middle) and near the exit hole (bottom). The sidewall roughness was higher with the higher repetition rate, the average Ra value being $0.2 \mu \mathrm{m}$ at $60 \mathrm{~Hz}$ and $0.16 \mu \mathrm{m}$ at 30 Hz. Roughness around the exit hole was less compared to the middle and upper areas. Further investigation is required to analyse fully the effect of laser parameters on sidewall roughness.

\subsubsection{Microcrack formation and other defects in microvias}

Since glass is brittle in nature it is likely to produce defects such as microcracks, chipping and undercuts along the edges and void formation along the sidewalls of the hole. Microcracking was a significant problem during microvia machining and was investigated using SEM observation. In most microvias, microcracks were confined to the sidewalls, however, the severity of cracking depended on the laser process parameters. In the microvias drilled at low repetition rates of the order of $20-30 \mathrm{~Hz}$, microcracks were confined to only a small area along the sidewalls. However, at the higher repetition rates of the order of $50-100 \mathrm{~Hz}$, microcracks were found along the whole range of thickness, and in some cases microcracks were extended onto the glass top and bottom surface through entry and exit holes. Figure 9 shows the SEM images of $100 \mu \mathrm{m}$ diameter microvias drilled at high repetition rates such as $100 \mathrm{~Hz}$ and 50 $\mathrm{Hz}$, highlighting the region of microcracks. Figures 9a and b show the microcracks along the sidewall through the thickness of the glass, while figure 9c shows the microcracks extended near the surface of the glass. 
It is difficult to predict the exact root cause of these cracks. Many theories have been mentioned in the literature by several authors regarding the behaviour of microcracking in glass. Lan et al. [23] has described microcracks induced during excimer laser machining as thermal in nature and has explained the mechanism of crack formation during machining. According to him the irradiated laser pulses caused excitation of electrons in the glass, which further dissipated excess energy into the lattice by generating phonons. If the laser pulse duration is in the nanosecond range as here (34 ns) then heat transfer from the hot electrons to the lattice plays a significant role to increase the temperature of the lattice and subsequently generate thermally induced stresses, which further cause microcracks in or near the ablated region. However, with increase in fluence even with a nanosecond pulse, the amount of excess heat dissipated in the surrounding area should reduce. This could be the case here since the amount of thermal damage around the entry and exit holes of microvias was found to reduce with increase in fluence. However, microcracks were still found in the sidewalls along the thickness of the glass in most microvias drilled with different combinations of fluence and repetition rate (high and low ranges of both parameters), which means that it is not possible to avoid thermal stresses entirely or that some other mechanism may also be involved in formation of microcracks in microvias. According to Keiper et al. [25] microcracks are formed during drilling of holes in glass due to pressure at the base of the hole which causes explosion of the strong plasma, but further suggested that they may also be due to thermally induced mechanical stresses in the glass. Further work is needed to investigate heat treatments that can remove the cracks or limit their effect on the mechanical properties of the glass. 
In addition to microcracks, voids were also observed in some microvias near the entry holes due to melting and ejection of the material. Figure 9a shows a void of around $2 \mu \mathrm{m}$ formed near the edge of the entry hole. Using very short drilling times of $5 \mathrm{~s}$ or less, at high fluence and repetition rates, such defects could be avoided.

Chipping and undercuts near the edges of the entry and exit holes were also observed in many microvias (figure 9d). However chipping and undercuts near the edges were avoided by using appropriate laser parameters such as high fluence and high repetition rates. Microvias produced at $4.5 \mathrm{~J} / \mathrm{cm}^{2}, 60 \mathrm{~Hz}$ repetition rate and $5 \mathrm{~s}$ irradiation time were of superior quality with minimum debris and recast around the holes.

As described above, a wide variation in the surface morphology of microvias and size and geometry of the exit holes was identified within a wide range of laser operating parameters. Fig 10 summarises the results for microvias with $100 \mu \mathrm{m}$ entry holes machined in a wide operating range based on the maximum fluence achievable at the work piece of $4.5 \mathrm{~J} / \mathrm{cm}^{2}$. This chart helps the selection of appropriate machining parameters for drilling microvias with a desired taper profile and exit hole diameter. From the overall investigation it was observed that microvias drilled at repetition rates of the order of $60 \mathrm{~Hz}$ and $5 \mathrm{~s}$ (300 pulses) and maximum fluence of $4.5 \mathrm{~J} / \mathrm{cm}^{2}$ had maximum exit hole diameter $(50 \mu \mathrm{m})$, minimum debris deposition and uniform side wall roughness.

\subsection{Microvia machining for glass substrate manufacture}

To investigate the application of the microvia machining process for electronics manufacture, a pattern of microvias to match a semiconductor flip-chip device was 
created with $100 \mu \mathrm{m}$ entry holes using the process window summarised in the previous section. A square pattern of microvias with a pitch size of $225 \mu \mathrm{m}$ and 300 $\mu \mathrm{m}$ in the horizontal and vertical direction was initially drilled in the $100 \mu \mathrm{m}$ thick glass using $3 \mathrm{~J} / \mathrm{cm}^{2}, 30 \mathrm{~Hz}$ and $30 \mathrm{~s}$ drilling time for each microvia which required 35 minutes to machine the whole pattern [26]. This could be reduced to 7 minutes by limiting the microvia drilling time to $5 \mathrm{~s}$ along with using higher fluence of $4.5 \mathrm{~J} / \mathrm{cm}^{2}$ and repetition rates of $50 \mathrm{~Hz}$ and above. For an actual industrial application, it would be possible to reduce the machining time for the same pattern further, to around 3 mins, by using an interchangeable mask with multiple holes (around 3 holes for 1 $\mathrm{mm}^{2}$ beam spot size) along with a more advanced excimer laser set-up capable of giving more fluence along with low divergence.

\section{Conclusion}

An excimer laser was used to successfully machine microvias in $100 \mu \mathrm{m}$ thick CMZ glass. $100 \mu \mathrm{m}$ diameter entry holes could be drilled in as little as $5 \mathrm{~s}$ with appropriate operating parameters and a process window was identified for microvia drilling. The fluence delivered to the workpiece was found to be very important and by modifying the beam delivery system, the fluence at the work piece was increased from $3 \mathrm{~J} / \mathrm{cm}^{2}$ to $4.5 \mathrm{~J} / \mathrm{cm}^{2}$. Increased fluence of $4.5 \mathrm{~J} / \mathrm{cm}^{2}$ led to reduced hole taper (taper angle of $14^{\circ}$ relative to the vertical) which enabled exit hole diameters up to 50 $\mu \mathrm{m}$ to be achieved in microvias with entry holes of $100 \mu \mathrm{m}$ diameter in $100 \mu \mathrm{m}$ thick glass. Process limits to drill the smallest microvias were explored and it was possible to drill through-hole microvias down to $40 \mu \mathrm{m}$ diameter in $50 \mu \mathrm{m}$ thick glass provided the maximum fluence at the work piece was $4.5 \mathrm{~J} / \mathrm{cm}^{2}$. 
Debris and recast layers formed around the holes, but it was possible to minimise debris deposition by laser drilling the glass after it was laminated with a photoresist layer. In addition, the surface roughness along the microvia sidewall varied with the laser machining parameters with average values between $0.16 \mu \mathrm{m}$ and $0.2 \mu \mathrm{m}$. Finally, microcracks were identified along the sidewalls of the $100 \mu \mathrm{m}$ diameter microvias drilled using a wide range of parameters. In general, it was found that using high fluence of $4.5 \mathrm{~J} / \mathrm{cm}^{2}$, with high repetition rate of the order of $50-60 \mathrm{~Hz}$ and short machining time of around 5 $-10 \mathrm{~s}$ gave the best quality of holes with minimum taper and reduced debris. The results demonstrate that it is possible to drill microvias in thin glass with high accuracy at dimensions suitable for electronic applications, but further work is needed to investigate the influence of features such as microcracking on the substrate reliability.

\section{Acknowledgements:}

The authors would like to acknowledge the EPSRC for funding this research project through the Innovative Electronics Manufacturing Research Centre (IeMRC) under original Grant Reference GR/T/07459/01. The authors would also like to thank Qioptiq for their technical support with special thanks to Mr James Hall.

\section{References:}

1. J. Fisher; Organic Interconnect Roadmap: Printed Circuit Design \& Fab, (2007)

2. C. W. Berlin, D. H. R. Sarma, W. A. Sozansky, D. W. Zimmerman; Proc. SPIE, Denver CO. 5231, 63 (2003)

3. S. Winkler, S. Berry; Chip Scale Review. (2005)

4. E. J. Vardaman; Adv Microelectronics, Texas: IMAPS. Vol. 30, 10 (2003)

5. P. Borgesen; Proc. SMTA, San Jose. 121 (1999)

6. K. Kandle; IPC Apex. (2009)

7. X. Cui, D. Bhatt, F. Khoshnaw, D.A. Hutt, P.P. Conway; Proc. $10^{\text {th }}$ IEEE EPTC, Singapore: 12 (2008)

8. L. Brusberg, H. Schröder, M. Töpper, N. Arndt-Staufenbiel, J. Röder, M. Lutz, H. Reichl; Proc. 59 ${ }^{\text {th }}$ IEEE ECTC, San Diego: 207 (2009) 
9. J. H. Lau, R. S. W. Lee; Microvias: Low Cost High Density Interconnects (McGraw-Hill, London, 2000)

10. H. Zheng, E. Gan, Gnian Cher Lim; J. Opt. Lasers Eng. 36, 355 (2001)

11. W. Hansen, P. Fuqua, F. Livingston, A. Huang, M. Abraham, D. Taylor, S. Janson, H. Helvajian; Ind. Phys. 8, 18 (2002)

12. M. Gower, N. Rizvi; Proc. HPLA: SPIE. 4065, 452 (2000)

13. B. Tan, K. Venkatakrishnan; J. Micromech. Microeng. 17, 1511 (2007)

14. Website. http://www.qioptiq.com/space.html

15. J.C. Ion; Laser Processing of Engineering Materials: Principles, Procedure and Industrial Application (Elsevier, Oxford, Butterworth-Heinemann, 2005)

16. R. Crafer, P.J. Oakley; Laser Processing in Manufacturing (Chapman \& Hall, London 1993)

17. D. Hulsenberg; Microstructuring of Glasses (Spinger, Berlin 2008)

18. Y. H. Chen, H. Y. Zheng, K. S. Wong, S. C. Tam; Proc. SPIE. 3184, 202 (1997)

19. H. Hornberger, R. Weissmann, N. Lutz; Glastech. Ber. Glass Sci. Technol. 69, 44 (1996)

20. Y. Liao, Y. chen, C. Chao, Y. Liu; Proc. SPIE. 5715, 110 (2005)

21. A. Bogaerts, Z. Chen; Spectrochim. Acta, Part B. 60, 1280 (2005)

22. D. Kawamura, A. Takita, Y. Hayasaki, N. Nishida; Appl. Phys A. 85, 39 (2006)

23. B. Lan, Ming-Hui Hong, Kai-Dong Ye, Zeng-Bo Wang, Shi-Xin Cheng, TowChong Chong; Appl. Phys. 43, 7102 (2004)

24. A. Tseng Y. Chen and K. Ma; J.Opt. Lasers Eng. 41, 827 (2004)

25. B. Keiper, H. Exner, U. Loschner, T. Kuntze; J. Laser Appl. 12, 189 (2000)

26. D. Bhatt, K. Williams, D. A. Hutt, P. P. Conway; Proc. $9^{\text {th }}$ IEEE EPTC, Singapore: 196 (2007)

\section{List of Figures:}

Figure 1: Excimer laser beam delivery system

Figure 2: Cross-section of a $100 \mu \mathrm{m}$ microvia drilled using $4 \mathrm{~J} / \mathrm{cm}^{2}$ fluence

Figure 3: Graphical presentation of the operating window for $100 \mu \mathrm{m}$ microvias machined in $100 \mu \mathrm{m}$ thick CMZ glass (a) Profile of exit hole diameter and irradiation time for different fluence and repetition rate (b) Profile of exit hole diameter and repetition rate at $3.0 \mathrm{~J} / \mathrm{cm}^{2}$ and $4.5 \mathrm{~J} / \mathrm{cm}^{2}$ fluence for fixed irradiation time of $20 \mathrm{~s}$ (c) Profile of exit hole diameter and number of pulses at $3.0 \mathrm{~J} / \mathrm{cm}^{2}$ and $4.5 \mathrm{~J} / \mathrm{cm}^{2}$ fluence for different repetition rates (d) Variation of taper angle with respect to energy fluence at $60 \mathrm{~Hz}$ repetition rate and $30 \mathrm{~s}$ irradiation time

Figure 4: SEM images of microvias machined in $100 \mu \mathrm{m}$ thick CMZ glass (a) As machined glass with loose and hard crust of debris (b) Machined glass after ultrasonic cleaning in an iso-propanol bath

Figure 5: $100 \mu \mathrm{m}$ microvias drilled in $100 \mu \mathrm{m}$ thick glass (a) $4.2 \mathrm{~J} / \mathrm{cm}^{2}, 50 \mathrm{~Hz}$ and $20 \mathrm{~s}$ (1000 shots) (b) $2.6 \mathrm{~J} / \mathrm{cm}^{2}, 60 \mathrm{~Hz}, 30 \mathrm{~s} \mathrm{(1800} \mathrm{shots)}$

Figure 6: Images of microvias drilled at $4.5 \mathrm{~J} / \mathrm{cm}^{2}$ fluence and $80 \mathrm{~Hz}$ repetition rate in glass laminated with a photoresist film (a) Optical image of microvia drilled with 400 pulses (b) Optical image of microvia drilled with1600 pulses (c) SEM micrograph of $100 \mu \mathrm{m}$ microvia drilled in photoresist laminated $50 \mu \mathrm{m}$ thick glass 
Figure 7: Optical micrographs of $100 \mu \mathrm{m}$ diameter microvias drilled at $4.5 \mathrm{~J} / \mathrm{cm}^{2}$ and $60 \mathrm{~Hz}$ repetition rate for different drilling times (a) and (b) Front and exit hole of microvia drilled for $5 \mathrm{~s}$ (c) and (d) Front and exit hole of microvia drilled for $10 \mathrm{~s} \mathrm{(e)} \mathrm{\&} \mathrm{(f)} \mathrm{Front} \mathrm{and} \mathrm{exit} \mathrm{hole} \mathrm{of} \mathrm{microvia} \mathrm{drilled} \mathrm{for} 20 \mathrm{~s}$

Figure 8: SEM images of $100 \mu \mathrm{m}$ diameter microvias drilled at the fluence of

$4.5 \mathrm{~J} / \mathrm{cm}^{2}, 50 \mathrm{~Hz}$ repetition rate and irradiation time of (a) $5 \mathrm{~s} \mathrm{(b)} 10 \mathrm{~s}$

(c) $20 \mathrm{~s}$

Figure 9: SEM micrograph of $100 \mu \mathrm{m}$ diameter microvia drilled in $100 \mu \mathrm{m}$ thick glass at $4.5 \mathrm{~J} / \mathrm{cm}^{2}$, (a) \&(b) at $100 \mathrm{~Hz}$ and $5 \mathrm{~s}$ (500 pulses), (c) at $50 \mathrm{~Hz}$ and $10 \mathrm{~s}$, (d) exit hole at $4.2 \mathrm{~J} / \mathrm{cm}^{2}, 40 \mathrm{~Hz} \& 20 \mathrm{~s}$.

Figure 10: Summary of operating window for $100 \mu \mathrm{m}$ diameter microvias drilled in $100 \mu \mathrm{m}$ thick CMZ glass, with respect to energy fluence, repetition rate and irradiation time (maximum fluence at the work piece of $4.5 \mathrm{~J} / \mathrm{cm}^{2}$ ) 


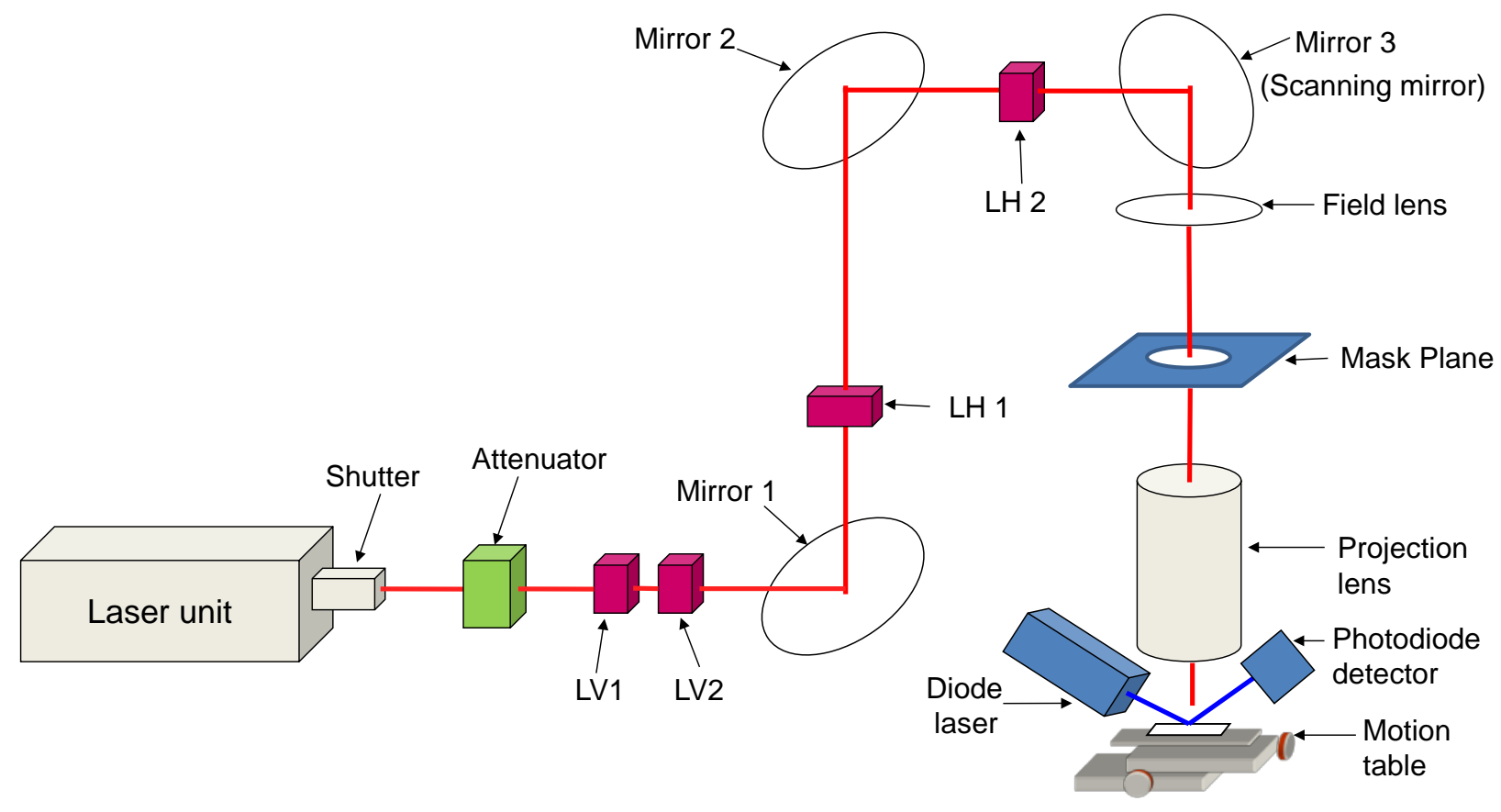

Fig 1. Excimer laser beam delivery system.

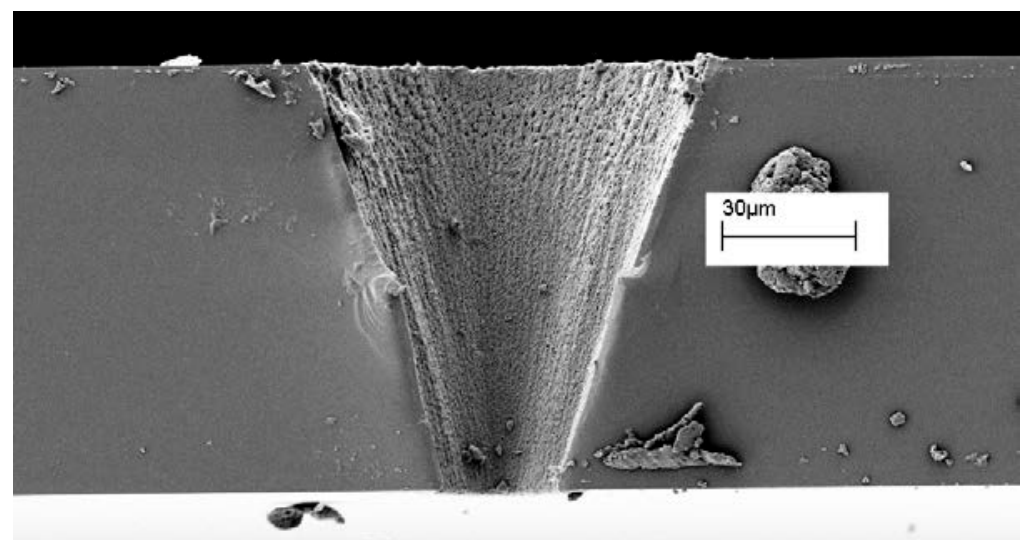

Fig 2. Cross-section of a $100 \mu \mathrm{m}$ microvia drilled using $4 \mathrm{~J} / \mathrm{cm}^{2}$ fluence. 


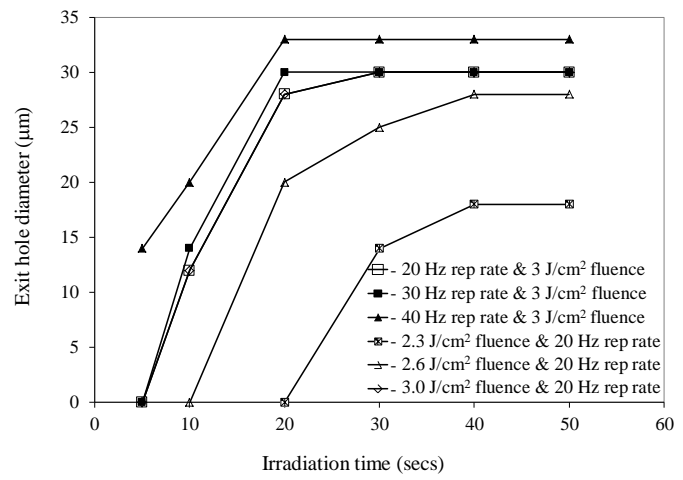

a

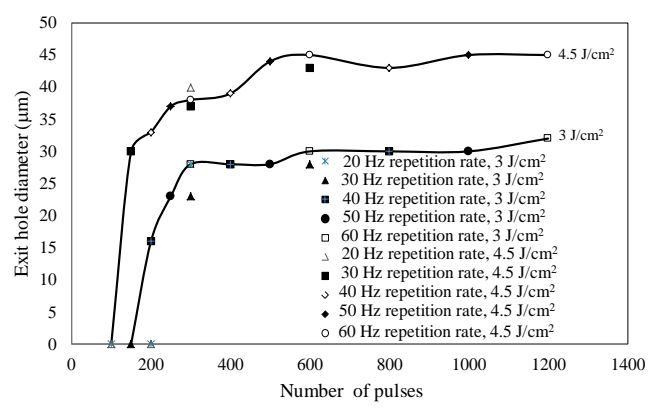

C

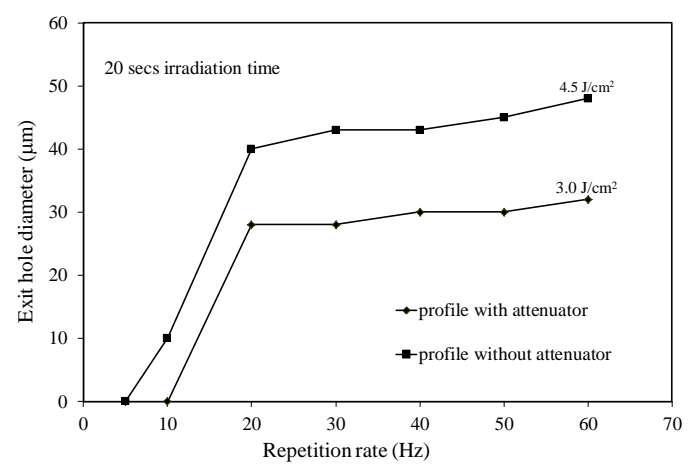

b

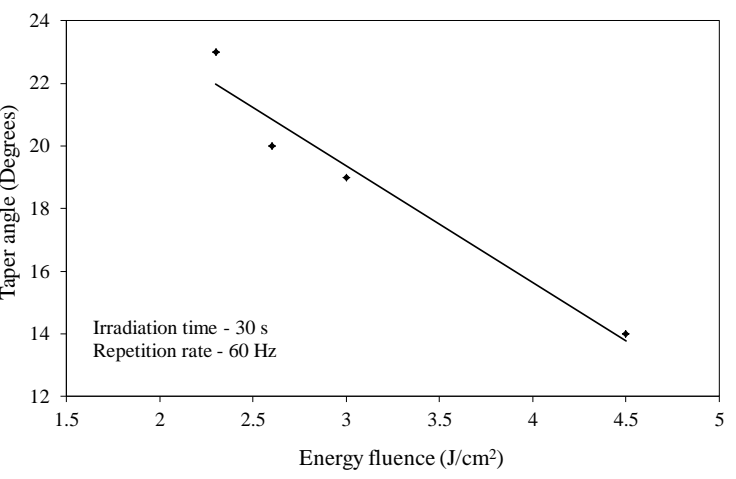

$\mathrm{d}$

Fig 3. Graphical presentation of the operating window for $100 \mu \mathrm{m}$ microvias machined in $100 \mu \mathrm{m}$ thick CMZ glass (a) Profile of exit hole diameter and irradiation time for different fluence and repetition rate.

(b) Profile of exit hole diameter and repetition rate at $3.0 \mathrm{~J} / \mathrm{cm}^{2}$ and $4.5 \mathrm{~J} / \mathrm{cm}^{2}$ fluence for fixed irradiation time of $20 \mathrm{~s}$. (c) Profile of exit hole diameter and number of pulses at $3.0 \mathrm{~J} / \mathrm{cm}^{2}$ and 4.5 $\mathrm{J} / \mathrm{cm}^{2}$ fluence for different repetition rates (d) Variation of taper angle with respect to energy fluence at $60 \mathrm{~Hz}$ repetition rate and $30 \mathrm{~s}$ irradiation time.
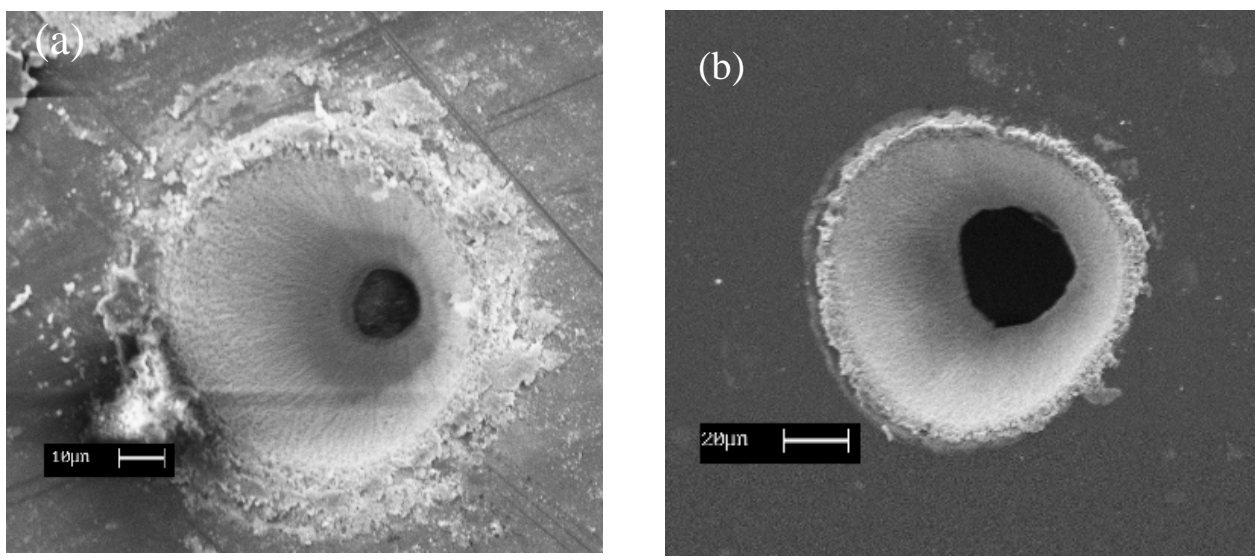

Fig 4. SEM images of microvias machined in $100 \mu \mathrm{m}$ thick CMZ glass. (a) as machined glass with loose and hard crust of debris, (b) machined glass after ultrasonic cleaning in an iso-propanol bath. 


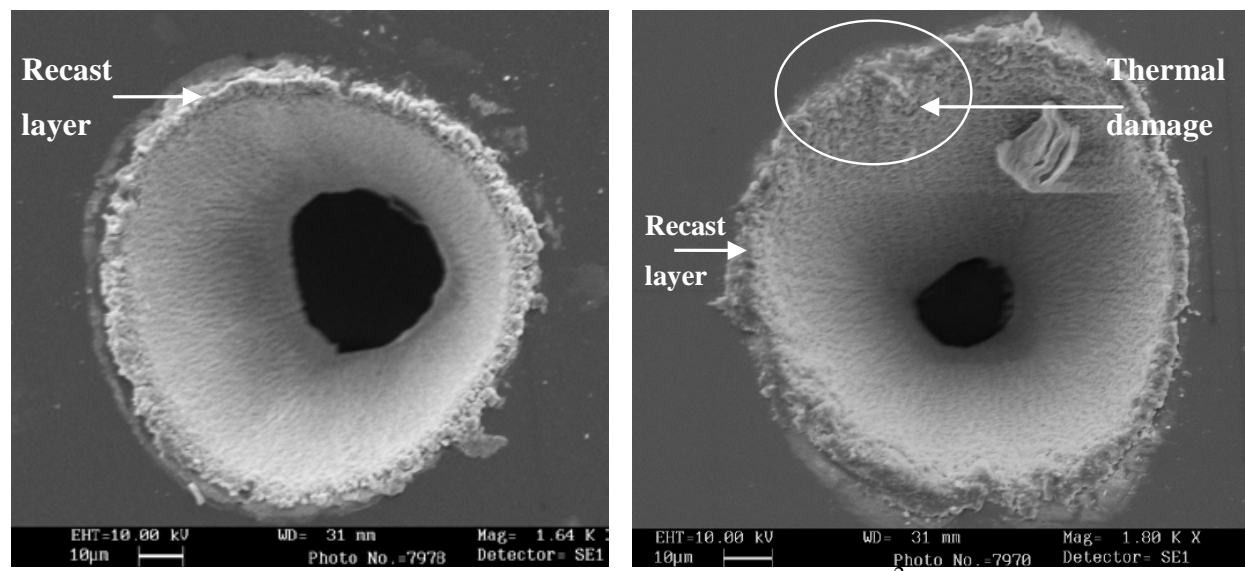

Fig 5. $100 \mu \mathrm{m}$ microvias drilled in $100 \mu \mathrm{m}$ thick glass at (a) $4.2 \mathrm{~J} / \mathrm{cm}^{2}, 50 \mathrm{~Hz}$ and $20 \mathrm{~s}$ (1000 shots) (b) $2.6 \mathrm{~J} / \mathrm{cm}^{2}, 60 \mathrm{~Hz}, 30 \mathrm{~s}$ (1800 shots).

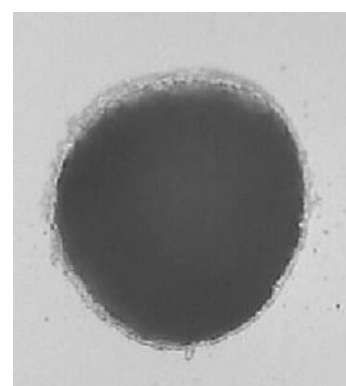

a

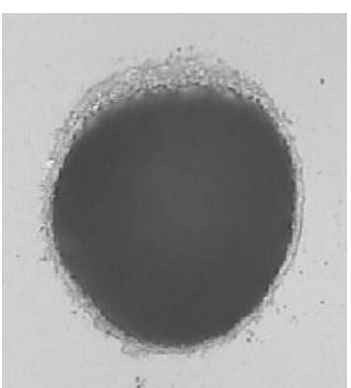

$\mathrm{b}$

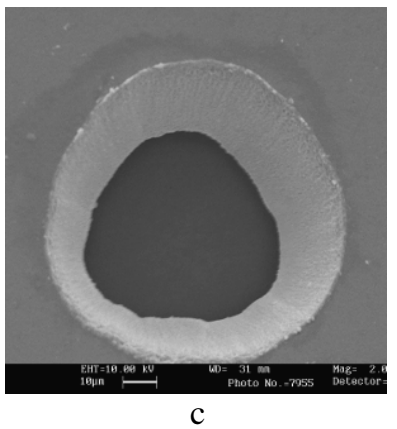

Fig 6. Images of microvias drilled at $4.5 \mathrm{~J} / \mathrm{cm}^{2}$ fluence and $80 \mathrm{~Hz}$ repetition rate in glass laminated with a photoresist film. Optical images: (a) 400 pulses, (b) 1600 pulses, (c) SEM micrograph of 100 $\mu \mathrm{m}$ microvia drilled in photoresist laminated $50 \mu \mathrm{m}$ thick glass. 


\section{Entry Hole}

\section{Exit Hole}

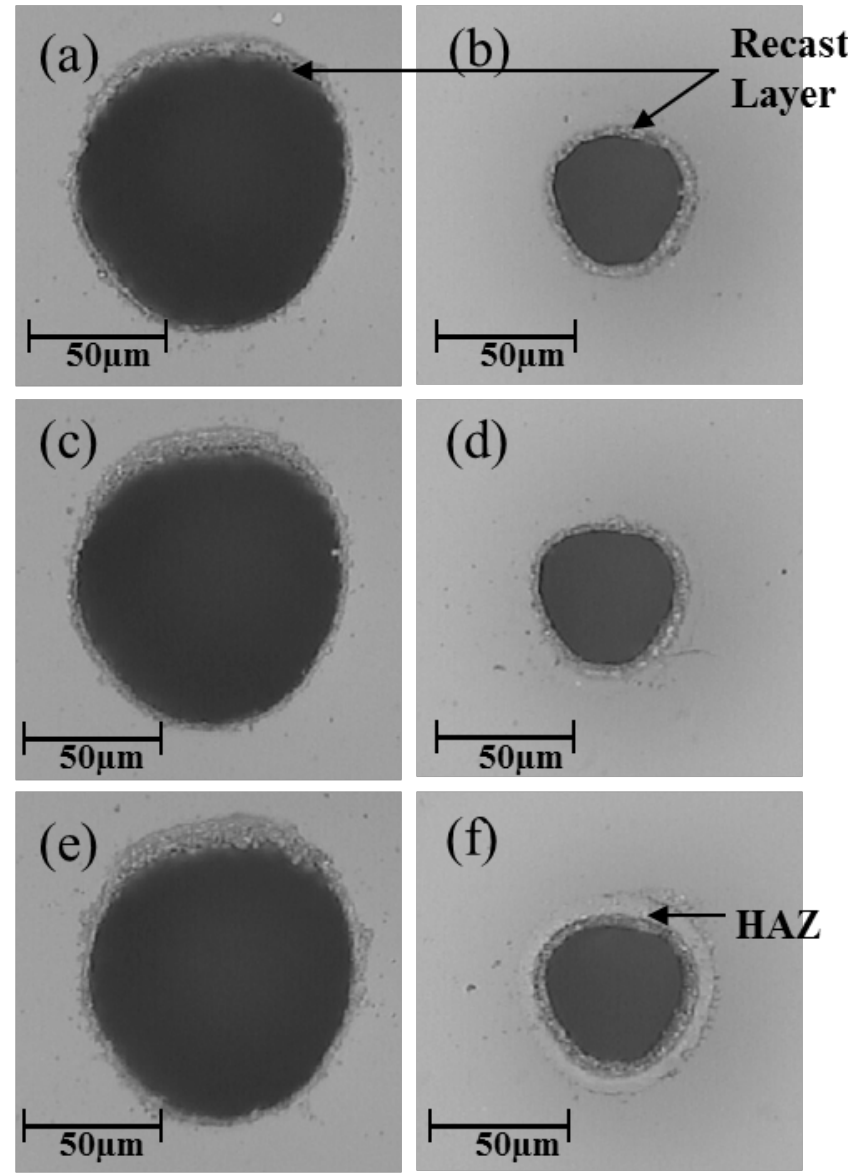

Fig 7. Optical micrographs of $100 \mu \mathrm{m}$ diameter microvias drilled at $4.5 \mathrm{~J} / \mathrm{cm}^{2}$ and $60 \mathrm{~Hz}$ repetition rate for different drilling times. (a) and (b) Front and exit hole of microvia drilled for 5 s, (c) and (d) Front and exit hole of microvia drilled for $10 \mathrm{~s} \mathrm{(e)} \mathrm{\&} \mathrm{(f)} \mathrm{Front} \mathrm{and} \mathrm{exit} \mathrm{hole} \mathrm{of} \mathrm{microvia} \mathrm{drilled} \mathrm{for} 20 \mathrm{~s}$.
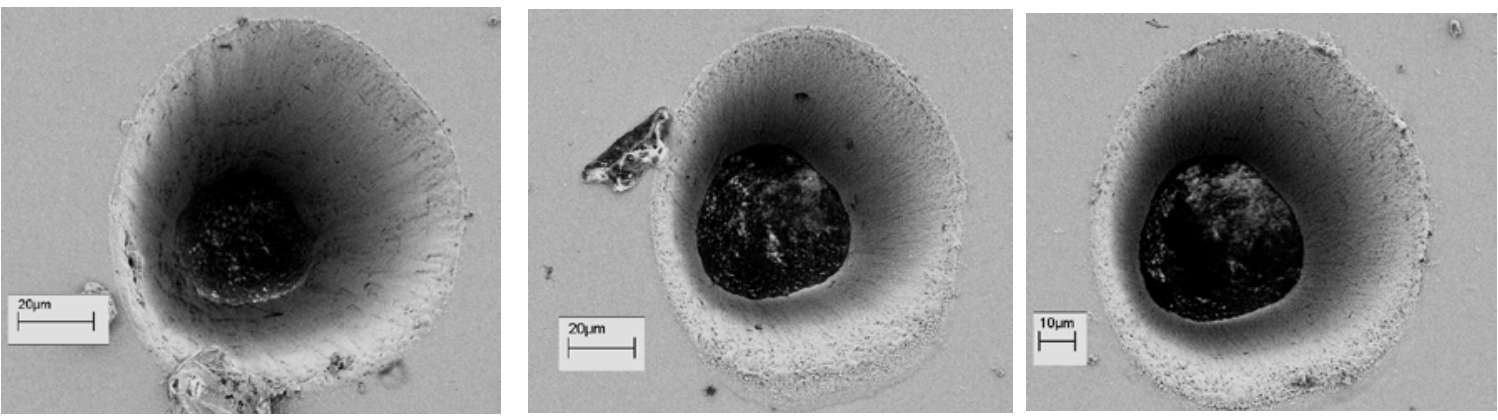

Fig 8. SEM images of $100 \mu \mathrm{m}$ diameter microvias drilled at a fluence of $4.5 \mathrm{~J} / \mathrm{cm}^{2}, 50 \mathrm{~Hz}$ repetition rate and irradiation time of (a) $5 \mathrm{~s} \mathrm{(b)} 10 \mathrm{~s} \mathrm{(c)} 20 \mathrm{~s}$. 

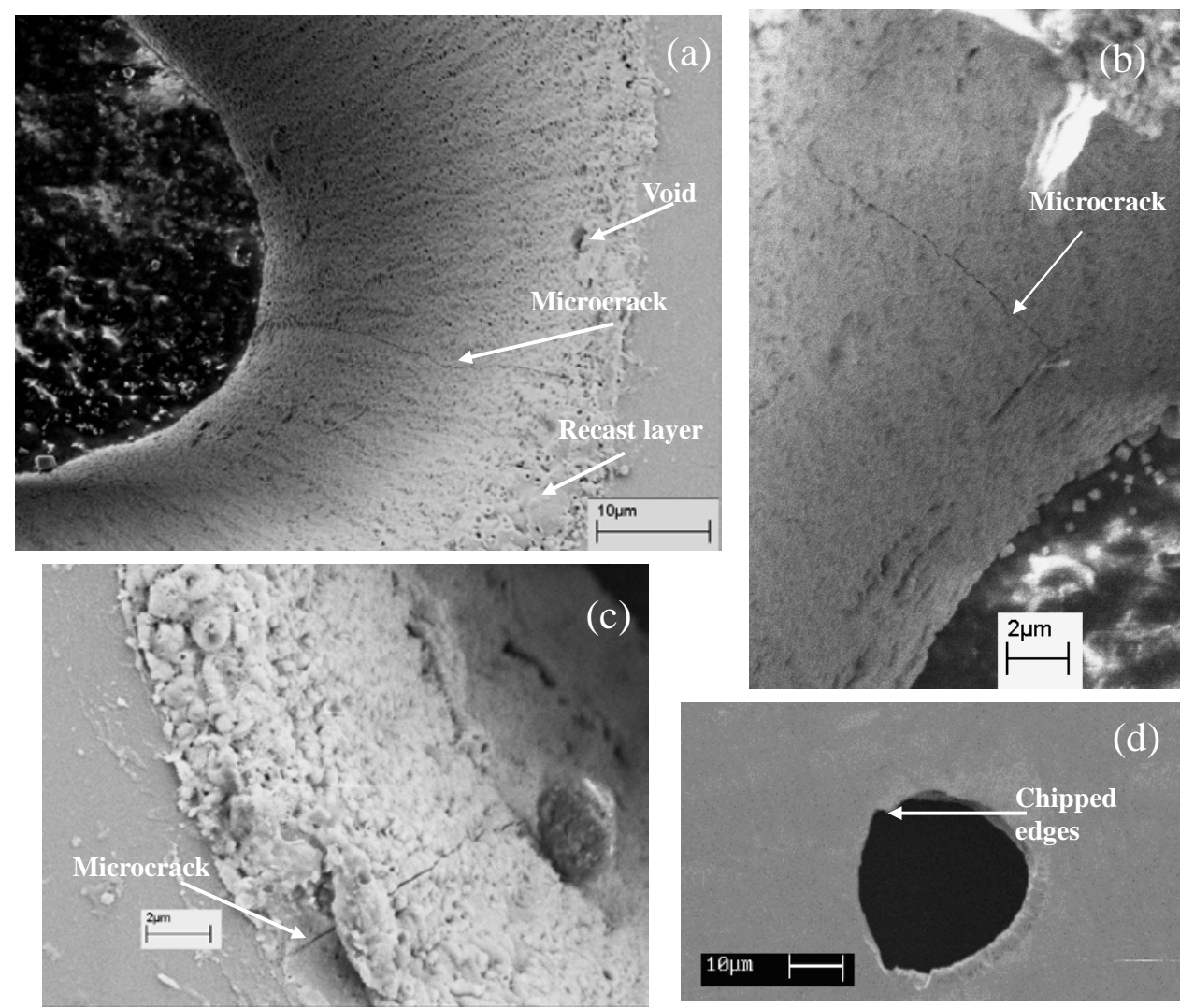

(c)

Fig 9. SEM micrograph of $100 \mu \mathrm{m}$ diameter microvia drilled in $100 \mu \mathrm{m}$ thick glass at $4.5 \mathrm{~J} / \mathrm{cm}^{2}$, (a) \&(b) at $100 \mathrm{~Hz}$ and $5 \mathrm{~s}$ (500 pulses), (c) at $50 \mathrm{~Hz}$ and $10 \mathrm{~s}$, (d) exit hole at $4.2 \mathrm{~J} / \mathrm{cm}^{2}$, $40 \mathrm{~Hz} \& 20 \mathrm{~s}$.

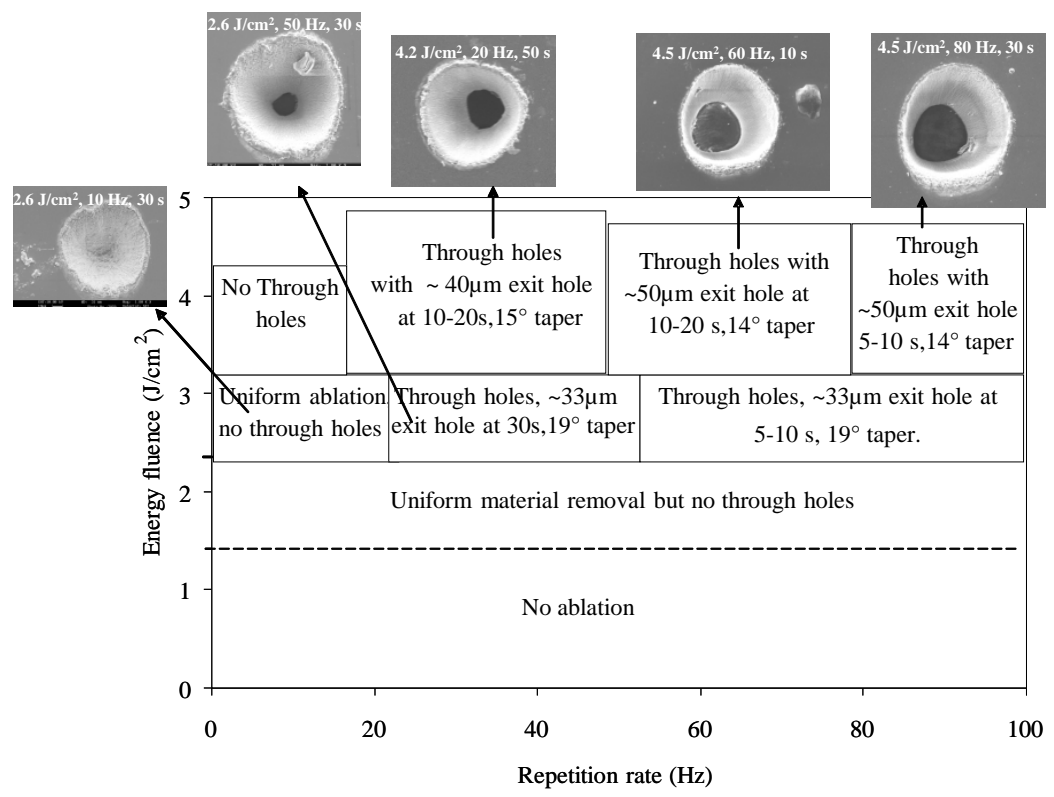

Fig 10. Summary of operating window for $100 \mu \mathrm{m}$ diameter microvias drilled in $100 \mu \mathrm{m}$ thick CMZ glass, with respect to energy fluence, repetition rate and irradiation time (maximum fluence at the work piece of $4.5 \mathrm{~J} / \mathrm{cm}^{2}$ ). 